\title{
International spinal cord injury endocrine and metabolic basic data set (version 1.2)
}

Spinal Cord (2012) 50, 567; doi:10.1038/sc.2012.37; published online 10 April 2012

In the article 'International spinal cord injury endocrine and metabolic basic data set" ${ }^{\text {' }}$ we have found omissions that we like to bring to the attention of your readers.

Under the variable heading of Gonadal status, the following sentence has now been inserted:

'In the female, puberty is defined as coincident with the initiation of breast development and/or with the appearance of axillary or pubic hair and before the start of menstruation; the term 'adult' should be applied if post-pubertal (that is, after the onset of menses) and not yet menopausal.

Correspondingly, on the data set form under the heading of Gonadal status for female, 'Adult' has been inserted.

For information we include the new data set form, but do also urge everyone to consult the website for all the updates of the International Spinal Cord Injury Data Sets:

http://www.iscos.org.uk/page.php?content $=20$
WA Bauman ${ }^{1}$, F Biering-Sørensen ${ }^{2}$ and A Krassioukov ${ }^{3}$ ${ }^{1}$ Veterans Affairs Rehabilitation Research \& Development Center of Excellence for the Medical Consequences of Spinal Cord Injury, James J. Peters Veterans Affairs Medical Center, Bronx, NY, USA; ${ }^{2}$ Clinic for Spinal Cord Injuries, NeuroScience Centre, Rigshospitalet, and Faculty of Health Sciences, University of Copenhagen,

Copenhagen, Denmark and

${ }^{3}$ International Collaboration On Repair Discoveries (ICORD),

Department of Medicine, University of British Columbia and Vancouver Coastal Health, Vancouver, British Columbia, Canada E-mail: william.bauman@va.gov

1 Bauman WA, Biering-Sørensen F, Krassioukov A. The international spinal cord injury endocrine and metabolic function basic data set. Spinal Cord 2011; 49: 1068-1072. 